BMJ Open

Sport \&

Exercise

Medicine

\title{
Mobility, proprioception, strength and FMS as predictors of injury in professional footballers
}

\author{
Jonathan Yeung, ${ }^{1}$ Andrew Cleves, ${ }^{2}$ Hywell Griffiths, ${ }^{3}$ Len Nokes ${ }^{1}$
}

To cite: Yeung J, Cleves A, Griffiths H, et al. Mobility, proprioception, strength and FMS as predictors of injury in professional footballers. BMJ Open Sport Exerc Med 2016;2: 0000134 doi:10.1136/bmjsem-2016000134

- Prepublication history for this paper is available online To view these files please visit the journal online (http://dx.doi.org/10.1136/ bmjsem-2016-000134).

Accepted 23 May 2016

\section{ABSTRACT}

Background: The premise of this study was to investigate if anthropometric variables such as mobility, proprioception, strength and modified Functional Movement Screen (mFMS) could be used as primary indicators of injury risk in an English Championship division football team. This study focused on moderate injuries occurring in the lower extremities, during the 2014/2015 competitive season.

Methods: To differentiate between minor, moderate and severe injuries, this study classified moderate injuries as an injury with an average injury severity of 2-28 days. This study is composed of 4 individual investigations. Each variable was assessed against 2 groups: injured $(n=6)$ and non-injured $(n=10)$. The 2 groups were compiled from the first team, with the criteria that each participant of this study required: full preseason assessment and injury history for the time period, 1 July 2014 to 19 March 2015. A MannWhitney $U$ test (0.05\% significance) was applied to statistically analyse if each variable showed any variation across the 2 groups. Effect size was estimated with Cliff's d.

Results: Strength asymmetry displayed significant difference ( $p=0.007)$, mobility, proprioception and mFMS did not $(p=0.263, p=0.792$ and $p=0.181$, respectively). Mean scores for mobility, proprioception, strength asymmetry and mFMS for injured versus non-injured players (effect size) were: 40.00 vs 38.00 (0.37), 10.33 vs $10.20(0.10), 61.13$ vs $30.40(0.80)$ and 7.33 vs $8.90(-0.4)$, respectively.

Conclusions: This study found no relationship between mobility/proprioception and injury risk; however, strength asymmetry was statistically significant in predicting injury and mFMS exhibited enough positive difference for recommendation of further investigation.

${ }^{1}$ Cardiff University, Cardiff, UK

${ }^{2}$ CEDAR, Cardiff Medicentre, University Hospital of Wales, Cardiff, UK

${ }^{3}$ Cardiff City Football Club, Cardiff, UK

Correspondence to Professor Len Nokes; Nokes@cardiff.ac.uk

\section{INTRODUCTION}

The risk of injury in a professional football player has been estimated to be 1000 times higher than for typical industrial occupations generally regarded as high-risk, ${ }^{1}$ with the most frequent injuries occurring in the lower limbs. $^{2}$ As lower limb injuries are so common, prevention of these injuries is important when considering personal grief,
New findings

- Strength asymmetry can be used as a predictor of lower limb injuries in footballers.

- Modified Functional Movement Screen potentially could be used as a predictor of lower limb injuries in footballers-however it is not a standardised test.

- Mobility and proprioception cannot be used as predictors of lower limb injuries in footballers.

disabling consequences and the high cost due of injuries ${ }^{3}$ in terms of treatment and absence from competitive play.

Professional footballers suffer 710 injuries per 100000 hours of training/match time, while $47 \%$ of players retire due to an injury-related problem. ${ }^{4}$

Teams that avoid injures tend to achieve better final league standings. ${ }^{5}$ A professional football club can expect around 50 injuries resulting in time lost from play each season. ${ }^{6}$ Both injury prevention strategies and rehabilitation after injury are complex, requiring specialist knowledge and experience of professional coaches and trainers. ${ }^{7}$

Though the effects of mobility, proprioception, strength asymmetry and Functional Movement Score (anthropometric variables) on injury have been studied previously, no study to date has incorporated all of these variables.

It has previously been found that mobility is related to injury in male athletes and that preseason mobility programmes could reduce injuries. ${ }^{8}$ These studies suggest that preseason and day-to-day mobility training could be beneficial in preventing injury. Modifications in flexibility training protocols have been found to reduce hamstring injuries in professional footballers, ${ }^{9}$ though the findings of this study were not fully conclusive due to variations in training patterns and not all clubs returning their survey. There 
has been some support that general joint laxity is predictive of leg injuries. ${ }^{3}$

Proprioception is defined as the sense of body motion - the ability to feel the body moving in space. ${ }^{10}$ It has been shown that an implementation of a proprioceptivecoordinative training programme increased all measured fitness indicators and resulted in a reduced injury incidence by $400 \%{ }^{11}$

The effectiveness of neuromuscular training in reducing the rate of knee injuries has also been evaluated: ${ }^{12}$ a 15 min neuromuscular warm-up programme reduced the overall rate of anterior cruciate ligament (ACL) injury in adolescent female football players by $64 \%$.

The effect of knee injury primary prevention programmes on ACL injury in female athletes in various sports has proven to be effective. ${ }^{13}$ This study found a $70 \%$ decrease in non-contact ACL injuries in the intervention group versus control group, attributable to prepractice warm-up type training protocols.

Strength asymmetry is considered a musculoskeletal abnormality, defined as a bilateral strength imbalance. ${ }^{14}$ It is important to understand that skills in football are unilateral and consist of patterns of asymmetrical movement ${ }^{15}$ as the majority of football players favour either their left or right leg, and that it is this preference that may cause asymmetry in strength in the lower limb. ${ }^{14}$

As the strength asymmetry score indicates differences in exerted force on an isometric hold, the lower the asymmetry scores the more balanced a player is, in terms of the left and right limb exerting equal force.

Strength asymmetry has previously been proposed as a possible identifier of injury risk ${ }^{16}$ and has been found to be negatively associated with injury in the lower extremities. ${ }^{7}$

Functional Movement Screen (FMS) is a seven-item (deep squat, hurdle step, in-line lunge, shoulder mobility, active straight-leg raise, trunk stability push-up and rotary stability) screen that assesses basic movement patterns ${ }^{17}$ and an athlete's mobility and stability. FMS was developed as a functional movement and dynamic balance assessment to capture the complex construct of motor control. Each item is scored from 0 to 3 for a total possible sum score of 21 points. Higher scores represent better functional movement. ${ }^{18}$ For this study, a modified version of FMS (mFMS) was used which better reflected the basic lower limb movement patterns, mobility and stability of a professional footballer.

Preparticipation data can be used to identify those whom may be at risk of injury. It can also be used to discover talent, advance skills and provide baseline data for return to sport after injury and to develop a profile which reflects the characteristics held by those participating in the sport. ${ }^{18-21}$

Currently functional movement tests and in particular the FMS as a screening tool is commonly used in sport. ${ }^{22}$ However, there is little conclusive evidence supporting the reliability and validity of the FMS. Furthermore, there is a lack of evidence to support the inclusion of the functional movement tests selected for the FMS and their relevance to screening tools for specific client groups.

Assessing basic fundamental movements through a more functional approach has the potential to focus on modifying sporting movement patterns rather than just concentrating on specific muscles or joints, ${ }^{20}{ }^{23}$ further support this by suggesting performance tests need to be designed to closely replicate the players' normal activities. In particular for football, this means including flexibility, movement patterns of main joints, specific football skills, power, speed and endurance. ${ }^{20}{ }^{24}$ Specific testing programmes should identify a player's physical profile revealing their strengths and weaknesses and thereby their injury susceptibility. ${ }^{25}$

FMS score has previously been shown to predict injury risk, even outside of a sporting setting ${ }^{26}$ and standardised intervention programmes have been shown to improve FMS scores. ${ }^{27}$

Studies have explicitly shown that players with low scores on the FMS scoring system were more likely to be injured than those with high scores. ${ }^{28}$

The aim of this study was to determine if mobility, proprioception, strength asymmetry and mFMS could be used as predictors of lower limb injury in professional footballers.

\section{METHODOLOGY}

Participants in this study were professional male footballers playing for an English Championship team during the 2014/2015 competitive season. Preseason assessments were performed for all players at the club $(n=31)$, including mobility, proprioception, strength asymmetry and mFMS. Injury data were recorded for participants between the 1 July 2014 and 19 March 2015. However, 15 players were discounted from the study due to transfers between clubs, leaving 16 players with a full preseason assessment and injury history.

The preseason assessment incorporates the entire body; however, this study is concerned with only the lower limb as the majority of injuries occur in the lower extremities. ${ }^{15}$ As part of the lower limb assessment, mobility was measured in 14 areas (box 1) and proprioception in 4 areas (box 2). Mobility and proprioception scores range from 0 to 4 , with 4 being perfectly executed and 0 representing extremely poor performance with pain experienced during execution attempt. Following previous methods, ${ }^{17} 19$ we used only the lower mobility/ proprioception score from either the left or right leg to contribute to the total score.

Strength asymmetry in each of the lower limbs was measured in four areas (box 3). A left versus right ratio was taken for each of the four strength areas. Total asymmetry was calculated as the sum of the four ratios.

The mFMS scoring system used in this study was a variation of the traditional seven-point test. This consisted of a four-point test scoring system in which the tests were: 


\section{Box 1 Mobility areas}

\section{Mobility}

- Ir/er@ 90/90,

- Hip Ext,

- Knee flexion,

- Passive SLR,

- Knee Fl,

- Knee Ext,

- Sup Tib/Fib,

- Ant Drawer 30,

- Drawer 90,

- Posterior Sag,

- With knee flexed,

- Ant Drawer with knee flexed,

Ankle valgus stress,

Knee to wall.

\section{Box 2 Proprioception areas}
Proprioception
- Valgus,
Varus,
- Hip posture,
Knee posture.

\section{Box 3 Strength areas}

\section{Strength \\ - Hamstring 30' BF bias, \\ - Hamstring 90' ST/SM, \\ - Long lever adduction, \\ Long lever abduction.}

overhead squat, overhead lunge, single leg squat, and hop and hold. Players performed each test three times, with the best result being recorded. Again, following previous methods, ${ }^{17} 19$ the lower score from either the left or right leg contributed to the total mFMS score.

Injury is defined as any injury sustained during football training session or first team match play that prevents a player from taking a full part in all training activities and/ or match play for one or more days after the time of injury. Injury severity is the number of days a player is injured. A player is deemed to be injured until he can undertake full training and be available for match selection. ${ }^{29}$

Game and training absences occurring as a result of illness were disregarded, and only moderate injuries sustained between 1 July 2014 and 19 March 2015 were included in the analysis. Moderate injury was based on, ${ }^{30}$ defined by resulting in a period of absence of between 2 and 28 days.

\section{STATISTICAL ANALYSIS}

Statistical analysis was performed with SPSS software (IBM). A Shapiro-Wilk test of the four continuous variables mobility, proprioception, strength and mFMS indicated that each of these variables was not normally distributed. The hypotheses that mean values of mobility, proprioception, strength and mFMS differed between injured and non-injured players were tested with the non-parametric Mann-Whitney $U$ test with a significance level of $\alpha=0.05$. In addition, the nonparametric Cliff's delta (d) was used to quantify effect size. Cliff's d was calculated by simple transformation of the Mann-Whitney U value. ${ }^{31}$ Cliff's d estimates that a value of an outcome measure (eg, mobility) for one player selected from the injured group is greater than the value for a player selected from the non-injured group, minus the reverse probability, a concept referring to the degree of overlap of the two sample distributions (injured and non-injured). Cliff's $d$ has a possible range from -1 to +1 ; values of \pm 1 indicate absence of overlap between groups whereas a value of 0 means the groups overlap completely. Values close to \pm 1 tend to occur when $p<0.05$ is observed. ${ }^{32}$ Effect size estimates are useful when the scale of measurement (and hence the size of difference between groups) is not easily understood.

\section{RESULTS}

Of the 16 players, 6 sustained a moderate lower limb injury and 10 remained uninjured. Of the four variables examined, only strength asymmetry was found to have a statistically significant difference between injured and noninjured players. Injured players were found to score 30.7 points higher for strength asymmetry than non-injured players ( 61.1 vs 30.4 , respectively, $\mathrm{p}=0.007$, table 1$)$. This is represented by an effect size of 0.8 . There was very little difference between injured and non-injured players for mobility score ( 40.0 vs 38.0 , respectively, $\mathrm{p}=0.263$, effect size 0.367 ), proprioception score (10.3 vs 10.2 , respectively, $\mathrm{p}=0.792$, effect size 0.1 ) and $\mathrm{mFMS}$ ( 7.3 vs 8.9 , respectively, $p=0.181$, effect size -0.433 ). The results suggest that of the four variables examined, only strength asymmetry shows potential as a predictor of injury.

\section{DISCUSSION}

Of the total injury incidence reported for 2014-2015 season, the majority were overuse injuries (9). Kicking/ striking/passing (6), running (6), jumping/landing (4) and stretching (1) all are believed to be linked to the functional movements identified in the mFMS.

Our study finding that scores for mobility, proprioception and mFMS did not differ substantially between injured and non injured players contrasts with the conclusions of previous studies. ${ }^{8}{ }^{12}{ }^{19}$ For mFMS, our study used a different threshold to define the group at high risk of injury $(\mathrm{FMS}<13)$, whereas previous studies have used an FMS score $<14$ to define the group at high risk of injury. Our more stringent definition of a high-risk group may have been a factor, although our observed 
Table 1 Points, Mann-Whitney U, significance and effect size results

\begin{tabular}{|c|c|c|c|c|c|c|}
\hline & Injured $(n=6)$ & Non-injured $(n=10)$ & Difference & Mann-Whitney U & Significance $p$ & Cliff's d \\
\hline Mobility & 40.00 & 38.00 & 2.00 & 41 & 0.263 & 0.367 \\
\hline Proprioception & 10.33 & 10.20 & 0.13 & 33 & 0.792 & 0.100 \\
\hline Strength asymmetry & 61.13 & 30.40 & 30.73 & 54 & 0.007 & 0.800 \\
\hline mFMS & 7.33 & 8.90 & 1.57 & 17 & 0.181 & -0.433 \\
\hline
\end{tabular}

difference in FMS between injured and non-injured players is small (1.6 scale points).

Otherwise it should be noted that our study sample size was limited, by practical constraints, to only 16 players which may result in inadequate statistical power to detect potentially important differences between injured and non-injured players. Published studies encountered have used a larger sample size $(n>60) .{ }^{27}$

Our observed differences in the mean strength asymmetry score between the injured and non-injured players is noticeably large (30.7 scale points), reflecting previous literature, ${ }^{16}$ in that the mean strength asymmetry score in injured players is approximately double to that of non-injured players. This finding is striking, though it should be noted that the analysis of four variables, each in turn, is subject to the limitation of repeated testing, whereby the risk of a type 1 error increases.

\section{SUMMARY}

Injuries in football occur due to numerous factors and can occur during matches or in training. Owing to the complexities and variation of these factors, efforts should be made towards a complete understanding of the key variables that separate a player from another. Mobility, proprioception and strength are such anthropometric variables that differ hugely between players, due to differences in size, shape and technical ability.

We investigated whether each of these four variables in turn differed significantly between two defined groups of English Championship players: those who had suffered injuries in the lower extremities and those who had not.

Of the four variables, strength asymmetry was the only variable that displayed a statistically significant difference between injured and non-injured players. Of the remaining three variables, none displayed any potential as a possible predictor of moderate injury risk. However, it should be noted that perhaps the study of mobility and proprioception in themselves is unnecessary because FMS incorporates both of these variables. This study would suggest in future investigations that the standardised FMS be used, thus allowing comparisons to other studies.

With regard to strength asymmetry, the results displayed significant difference between injured and noninjured players; thus, this study can recommend that strength asymmetry could be used as a predictor of injury.
Funding By Cardiff University, 10.13039/501100000866.

Competing interests None declared.

Ethics approval Cardiff University Ethical Committee.

Provenance and peer review Not commissioned; externally peer reviewed.

Open Access This is an Open Access article distributed in accordance with the Creative Commons Attribution Non Commercial (CC BY-NC 4.0) license, which permits others to distribute, remix, adapt, build upon this work noncommercially, and license their derivative works on different terms, provided the original work is properly cited and the use is non-commercial. See: http:// creativecommons.org/licenses/by-nc/4.0/

\section{REFERENCES}

1. Hawkins RD, Hulse MA, Wilkinson $C$, et al. The association football medical research programme: an audit of injuries in professional football. Br J Sports Med 2001;35:43-7.

2. Wong $\mathrm{P}$, Hong $\mathrm{Y}$. Soccer injury in the lower extremities. $\mathrm{Br} \mathrm{J}$ Sports Med 2005;39:473-82.

3. Dallinga JM, Benjaminse A, Lemmink KA. Which screening tools can predict injury to the lower extremities in team sports? Sports Med 2012;42:791-815.

4. Drawer S, Fuller CW. Evaluating the level of injury in English professional football using a risk based assessment process. $\mathrm{Br}$ $J$ Sports Med 2002;36:446-51.

5. Arnason A, Sigurdsson SB, Gudmundsson A, et al. Physical fitness, injuries, and team performance in soccer. Med Sci Sports Exerc 2004;36:278-85.

6. Ekstrand J, Hägglund M, Waldén $M$, et al. Injury incidence and injury patterns in professional football: the UEFA injury study. $\mathrm{Br} J$ Sports Med 2009;45:553-8.

7. Grygorowicz M, Kubacki J, Pilis W, et al. Selected isokinetic tests in knee injury prevention. Biol Sport 2010;27:47-51.

8. Krivickas LS, Feinberg JH. Lower extremity injuries in college athletes: relation between ligamentous laxity and lower extremity muscle tightness. Arch Phys Med Rehabil 1996;77:1139-43.

9. Dadebo B, White J, George KP, et al. A survey of flexibility training protocols and hamstring strains in professional football clubs in England. Br J Sports Med 2004;38:388-94.

10. Batson $\mathrm{G}$. Update on proprioception considerations for dance education. J Dance Med Sci 2009;13:35-7.

11. Knobloch K, Martin-Schmitt S, Gösling T, et al. Prospective proprioceptive and coordinate training for injury prevention in elite female soccer. Sportverletz Sportschaden 2005;19:123-9.

12. Walden M, Atroshi I, Magnusson $\mathrm{H}$, et al. Prevention of acute knee injuries in adolescent female football players: cluster randomised controlled trial. BMJ 2012;344:1-11.

13. Michaelidis M, Koumantakis GA. Effects of knee injury primary prevention programs on anterior cruciate ligament injury rates in female athletes in different sports: a systematic review. Phys Therapy Sport 2014;15:200-10.

14. Daneshjoo A, Rahnama N, Mokhtar AH, et al. Bilateral and unilatera asymmetries of isokinetic strength and flexibility in male young professional soccer players. J Hum Kinet 2013;36:45-53.

15. Fousekis K, Tsepis E, Vagenas G. Lower limb strength in professional soccer players: profile, asymmetry, and training age. J Sports Sci Med 2010;9:364-73.

16. Schache AG, Crossley KM, Macindoe IG, et al. Can a clinical test of hamstring strength identify football players at risk of hamstring strain? Knee Surg Sports Traumatol Arthrosc 2010;19:38-41.

17. Bradberry DR. Strength, flexibility, functional movement and injury in collegiate men football players. University of Georgia, 2010:1-30.

18. Cook G, Burton L, Hoogenboom B, et al. Pre-participation screening the use of fundamental movements as an assessment of functionpart 1. N Am J Sports Phys Ther 2006a;1:62-70. 
19. Cone J. Soccer-specific performance testing of fitness and athleticism: the development of a comprehensive player profile. Strength Cond J 2012;35:11-19.

20. Rosch D, Hodgson R, Peterson TL, et al. Assessment and evaluation of football performance. Am J Sports Med 2000;28:29-39.

21. Cook G, Burton L, Hoogenboom B, et al. Pre-participation screening: the use of fundamental movements as an assessment of function-part 2. N Am J Sports Phys Ther 2006b;1:132-9.

22. Cook G. Athletic body in balance: optimal movement skill and conditioning for performance. Human Kinetics, 2003.

23. Schneiders A, Davidsson A, Hörman E, et al. Functional movement screen normative values in a young, active population. Int J Sports Phys Ther 2010;6:75-80.

24. Requena B, González-Badillo JJ, de Villareal ES, et al. Functional performance, maximal strength, and power characteristics in isometric and dynamic actions of lower extremities in soccer players. J Strength Cond Res 2009;23:1391-401.

25. Balsom P. Evaluation of physical performance. Handbook of Sports Medicine and Science-Football (Soccer). Oxford: Blackwell Scientific Publications, 1994:102-23.
26. O'Connor FG, Deuster PA, Davis J, et al. Functional movement screening: predicting injuries in officer candidates. Med Sci Sports Exerc 2011;43:2224-30.

27. Kiesel K, Plisky P, Butler R. Functional movement test scores improve following a standardized off-season intervention program in professional football players. Scand J Med Sci Sports 2009;21:287-92.

28. Kiesel K, Plisky PJ, Voight ML, et al. Can serious injury in professional football be predicted by a preseason functional movement screen. N Am J Sports Phys Ther 2007;2:147-58.

29. The Football Association. The Football Association Injury and IIIness Surveillance Study. The Champions League 2014-15. 2015.

30. Hägglund M, Waldén M, Bahr R, et al. Methods for epidemiological study of injuries to professional football players: developing the UEFA model. Br J Sports Med 2005;39:340-6.

31. Norman C. Dominance statistics: ordinal analyses to answer ordinal questions. Psychol Bull 1993;114:494.

32. Macbeth G, Razumiejczyk E, Ledesma R, et al. Cliff's delta calculator: a non-parametric effect size program for two groups of observations. Univ Psychol 2011;10:545-55. 